\title{
Molecular recognition by cyclophane hosts
}

\author{
S. B. Ferguson, E. M. Seward, E. M. Sanford, M. Hester, M. Uyeki, and F. Diederich* \\ Department of Chemistry and Biochemistry, University of California at Los Angeles, Los \\ Angeles, California 90024 - 1569
}

\begin{abstract}
Synthetic cyclophane hosts form highly structured complexes with aromatic substrates in aqueous and organic solutions. Solvation-desolvation processes are recognized as a central factor that determines the stability of these complexes. From comparative studies it is evident that the driving force for complexation is much larger in aqueous solutions than in organic solvents. Investigations into the nature of the special driving force for molecular complexation in aqueous solution show that the binding of neutral benzene guests in deep, apolar cyclophane cavities is predominantly enthalpically driven and that the entropic contributions are very unfavorable. The nature of this enthalpic hydrophobic effect is discussed in terms of an increase in cohesive interactions between water molecules and in attractive dispersion interactions upon complexation. Binding studies in a series of organic solvents covering the entire polarity range demonstrate that the complexation strength is highly solvent dependent. Differences in complex stability are discussed in terms of the ability of the solvent to compete effectively with the guest for the cavity binding site. The different solvation requirements of cation versus apolar binding are demonstrated with a novel ditopic host. By changing from a water/methanol (60/40) mixture to pure methanol, this host can be switched from an efficient binder of neutral aromatics to a good host for potassium cations.
\end{abstract}

\section{INTRODUCTION}

Analyses of enzyme and antibody binding sites find highly preorganized hydrophobic pockets formed by peptide backbones which provide a suitable environment for complexing apolar organic substrates. Binding of these substrates by macrocyclic compounds with enforced binding cavities should involve the same driving forces that exist in biological systems. Studies of model systems provide an efficient method for investigating the contributions of specific forces and interactions that control biotic and abiotic complexation phenomena.

Investigations involving the cyclodextrins ${ }^{[1]}$ and synthetic cyclophane hosts $[2,3]$ have shown that stable complexes with apolar organic substrates can form in aqueous solutions. The highly structured complexes of cyclophanes with neutral arenes in water can approach enzyme-substrate complexes in their stability $\left(\mathrm{K}_{\mathrm{a}}>10^{6} \mathrm{~L} \cdot \mathrm{mol}^{-1}\right)$. ${ }^{[4]}$ Inclusion complexation between neutral aromatic guests and cyclophane hosts also occurs in a variety of organic solvents. ${ }^{[5]}$ From comparative binding studies, it is evident that the complexation of neutral arenes is more efficient in aqueous solution than in organic solvents. In this account, three studies are presented that demonstrate the central role of solvation for apolar host-guest interactions. The first investigation provides evidence for a strong enthalpic hydrophobic effect which promotes complexation of neutral benzene guests by novel cyclophane hosts. [6] The second study demonstrates that the strength of host-guest binding interactions in organic solvents of different polarities is determined primarily by the ability of the solvent molecules to compete with the guest for the cavity binding site. The third study shows how a change in solvent switches a novel ditopic cyclophane host from a good cation binder to an efficient receptor for neutral naphthalene derivatives. [7]

\section{A STRONG ENTHALPIC HYDROPHOBIC EFFECT IN AQUEOUS SOLUTION}

We prepared the octamethoxy-substituted cyclophane hosts 1 and 2 to analyze in temperature-dependent binding studies the thermodynamic characteristics for aqueous solution complexation of neutral benzene derivatives. [6] The eight methoxy substituents have a profound effect on the shape of the binding sites, the aggregation characteristics, and the binding capabilities of the two cyclophanes. The X-ray structure of 1 (Figure 1) as a diiodide shows a very deep, preorganized cavity with the methoxy groups aligned in the planes of the phenyl rings. The depth of the cavity in 1 and 2 is almost doubled as compared to analogous cyclophane hosts ${ }^{8]}$ with hydrogen atoms instead of the methoxy groups. The distance between the methyl hydrogen atoms of meta-methoxy groups in 1 is $\approx 8 \AA$, almost twice as large as the $\approx 4.2 \AA$ separation between two meta-hydrogens. The methoxy groups also make the aromatic cavity walls more polarizable. 


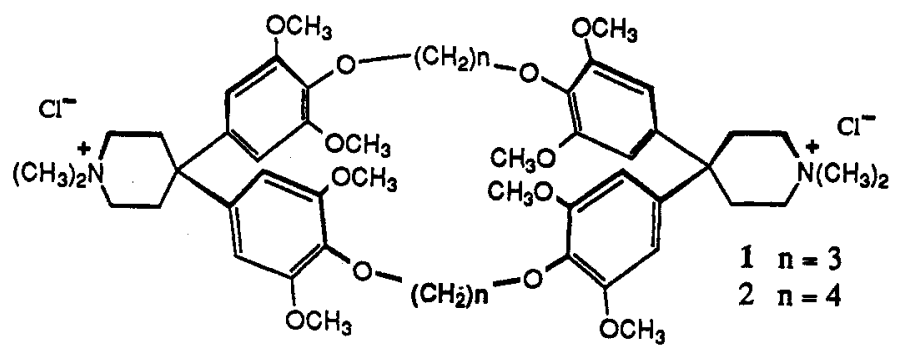

The addition of methoxy groups substantially reduces the aggregation tendencies of the charged hosts. Macrocycles 1 and 2 have critical aggregation concentrations (CAC's) greater than $1.0 \times 10^{-2} \mathrm{~mol}^{-\mathrm{L}^{-1}}$ while similar macrocycles without methoxy substituents have CAC's of $2.5 \times 10^{-3}$ and $1.6 \times 10^{-4} \mathrm{~mol} \cdot \mathrm{L}^{-1}$ respectively. ${ }^{[9]}$ The large CAC's provided optimal experimental conditions for a van't Hoff analysis of temperature dependent binding data. At the endpoints of ${ }^{1} \mathrm{H} N M R$ titrations with [host] $\approx 1 \times 10^{-2} \mathrm{~mol} \cdot \mathrm{L}^{-1}$ and [guest] $\approx 5 \times 10^{-4} \mathrm{~mol} \cdot \mathrm{L}^{-1}$ in water, we observed resonances that indicated greater than $98 \%$ complexation of the guest. Binding titration data that represents $\approx 40-98 \%$ complexation of the guest provided very accurate association constants which are needed to obtain significant values from a van't Hoff analysis.

Table 1 shows the association constants $K_{\mathrm{a}}(293 \mathrm{~K})$ for selected complexes of 1 and 2 with neutral para-substituted benzene guests in water. These $K_{\mathrm{a}}$ values, especially those for the complexes of macrocycle 2 , are extraordinarily large. The analysis of ${ }^{1} \mathrm{H}$ NMR complexation shifts suggests that the complexed guests are axially oriented presumably adopting a position in the cavity which favors both $\pi-\pi$ stacking and edge-to-face aryl- $\mathrm{H}_{\text {guest }} \cdots \pi-$ aryl ${ }_{\text {host }}$ interactions between the two binding partners. ${ }^{[3,8]}$ The contribution of electron donor-acceptor (EDA) interactions, observed in previous studies in aqueous and organic solutions, $[3,10,11]$ is surprisingly not obvious for complexes of 1 and 2 in aqueous solution (Table 1). These interactions may be masked by differences in the solvation and the hydrophobicity of the various benzene substrates. The various benzene derivatives differ considerably in their Hansch partition coeffients. ${ }^{[12]}$

Table 1: Association constants $K_{\mathrm{a}}$, enthalpic $\left(\Delta H^{\circ}\right)$, and entropic $\left(T \Delta S^{\circ}\right)$ contributions to the free energies of complexation $\left(\Delta G^{\circ}\right)$ at $293.4 \mathrm{~K}$ for complexes of hosts 1 and 2 with 1,4-disubstituted benzene guests in $\mathrm{D}_{2} \mathrm{O}$ and methanol- $d_{4}$

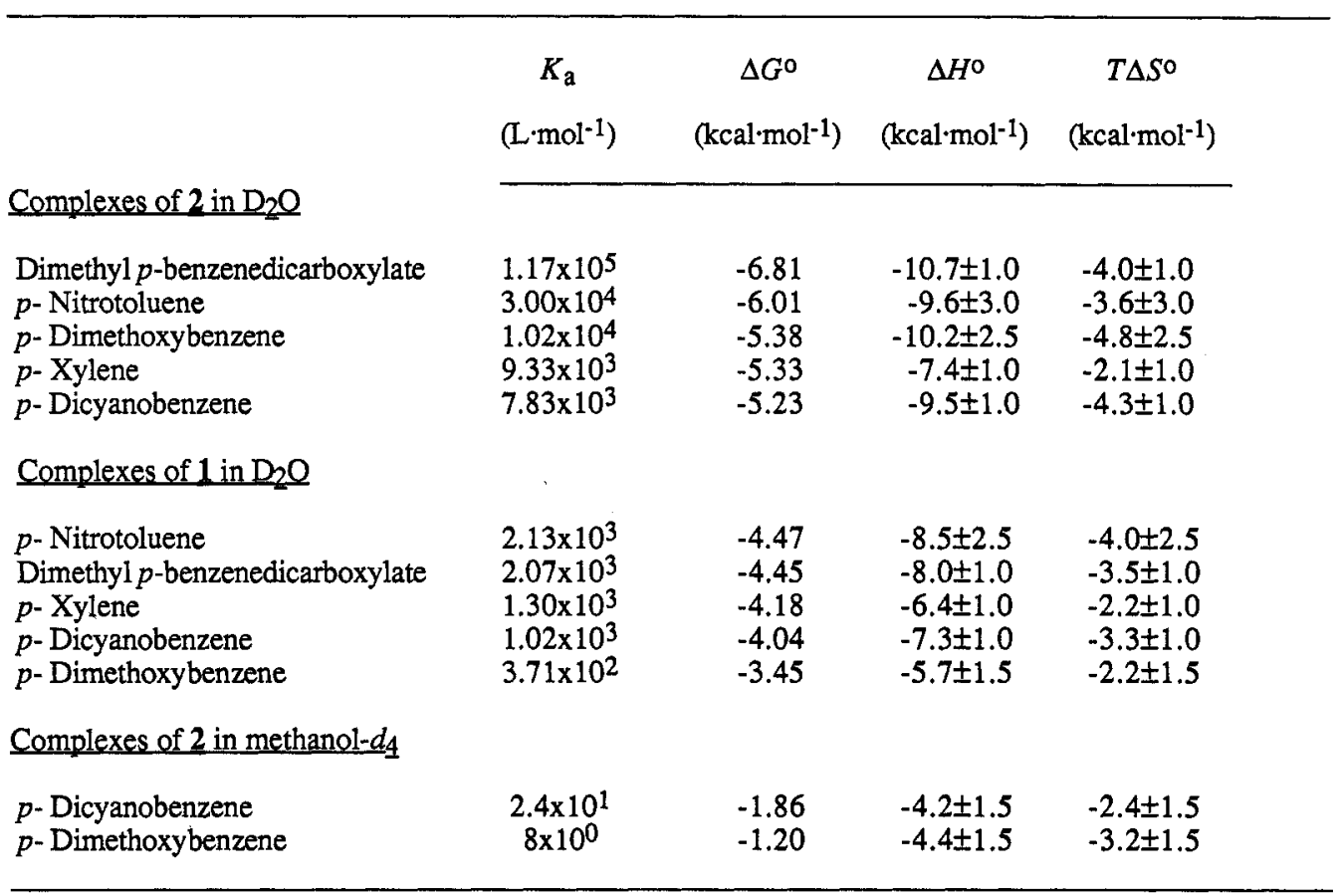


Table 1 shows that the complexes of 2 with $p$-dimethoxybenzene and $p$-dicyanobenzene in methanol- $d_{4}$ are $3-4$ $\mathrm{kcal} \cdot \mathrm{mol}^{-1}$ (at $293 \mathrm{~K}$ ) less stable than the corresponding complexes formed in aqueous solution. This data is representative of studies involving various benzene guests and demonstrates a special driving force for complexation which exists only in aqueous solution.

The van't Hoff analysis of temperature-dependent studies provided the thermodynamic quantities $\Delta H^{\circ}$ and $T \Delta S^{\circ}$ shown in Table 1. A rather narrow temperature range of $\approx 25^{\circ}$ was chosen to avoid non-linearity of van't Hoff plots resulting from possible heat capacity changes. All complexes studied in aqueous solution demonstrate a favorable enthalpic contribution and a large unfavorable entropic contribution to the free energy of complexation. Complexation of benzene derivatives with our hosts in aqueous solution is predominantly enthalpically driven.[13,14] A comparison of enthalpic and entropic contributions for binding in water versus methanol demonstrates a very large favorable enthalpic driving force that is only present in aqueous solution. The analysis of the ${ }^{1} \mathrm{H}$ NMR complexation shifts shows that the geometry of the complexes in water and methanol is approximately the same. Hence, the observed differences in enthalpic contributions presumably do not result from differences in attractive interactions betwen host and guest. Much of the favorable enthalpic component must result from specific contributions of water. We have identified two components of this strong enthalpic hydrophobic effect: changes in cohesive interactions of water and changes in dispersion interactions of the system. Water molecules that solvate the free guests and especially the cavity of the free host have reduced cohesive interactions and are enthalpically higher in energy than water molecules in the bulk solvent. The X-ray crystal structure of 1 shows two disordered water molecules located deep in the cavity. Water molecules in the binding site are presumably participating in fewer strong hydrogen bonds than bulk solvent molecules. Upon inclusion complexation, these water molecules are released into the bulk and become enthalpically lower in energy. Saenger has suggested that the release of "high-energy water" from the cavity into the bulk solvent represents one of the major driving forces for cyclodextrin complexation. ${ }^{[15]}$

Another component of the enthalpic hydrophobic effect involves the low polarizability of water molecules as compared to hydrocarbon material. $[16,17] M c$ Cammon, Wolynes, and Karplus in their molecular dynamics simulations [18] introduced as nonbonded parameters the polarizabilities $\alpha=1.20 \AA^{3}$ for an OH-residue, $1.35 \AA^{3}$ for an aliphatic $\mathrm{CH}, 1.77 \AA^{3}$ for an aliphatic $\mathrm{CH}_{2}, 2.17 \AA^{3}$ for a methyl group and $2.07 \AA^{3}$ for an aromatic $\mathrm{CH}$ group. The attractive $B$ term in the " $A r^{-12}-B r^{6}$ " Lennard-Jones potential to define van der Waals interactions can be calculated from the Slater-Kirkwood equation and is proportional to the polarizability $\alpha$ of the interacting atoms. Dispersion energies are additive, and at constant distances between interacting atoms, the attractive forces increase with increasing polarizability of atoms. Due to the low polarizability of oxygen atoms and the hydroxyl residue, the dispersion forces between water molecules and an apolar surface are weaker than the forces between two apolar organic surfaces. Upon formation of inclusion complexes of 1 and 2 , the attractive dispersion forces increase since the contacts between water molecules and the hydrocarbon surfaces of host and guest are replaced by close contacts between the surfaces of the two binding partners. The significant decrease in the enthalpic driving force in methanol solutions results because methanol possesses a polarizable methyl group that interacts more favorably with free host and guest than water does. Therefore, the differences in dispersion interactions between the nonbinding and the binding state of a host and guest in methanol are significantly reduced.

Our cyclophanes represent interesting models for the hydrophobic pocket binding sites in enzymes and antibodies. A literature survey shows that enzyme or antibody binding to aromatic substrates (Table 2) is defined by thermodynamic characteristics that are similar to those shown in Table 1 for the complexation by 1 and 2 . In almost all reported studies with aromatic substrates, both enthalpic and entropic contributions are very negative. Although hydrogen bonding also contributes to the biological recognition of the substrates shown in Table 2, a large part of the favorable enthalpic parameters certainly can be explained by the enthalpic hydrophobic effect described above. Studies with other receptors are now under way in our laboratory to determine whether the classical hydrophobic effect, [19] having thermodynamic characteristics $\Delta H^{\circ} \approx 0, T \Delta S^{\circ}>0$, and $\Delta C_{\mathrm{p}}{ }^{\circ}<0$, is observed when the hydrophobic surface areas of the binding site and of the guest are increased.[20]

Table 2: Thermodynamic characteristics for biological recognition of aromatic substrates at $298 \mathrm{~K}$ [21]

\begin{tabular}{lllll}
\hline \multicolumn{1}{c}{ Receptor } & Substrate & $\Delta G^{\circ}\left(\mathrm{kcal}^{\mathrm{m}} \mathrm{mol}^{-1}\right)$ & $\Delta H^{\circ}\left(\mathrm{kcal}^{\left.-\mathrm{mol}^{-1}\right)}\right.$ & $T \Delta S^{\circ}\left(\mathrm{kcal} \cdot \mathrm{mol}^{-1}\right)$ \\
\hline$\alpha$-chymotrypsin & proflavin & -6.0 & -11.3 & -5.4 \\
$\alpha$-chymotrypsin & indole & -4.3 & -15.2 & -11.0 \\
$\alpha$-chymotrypsin & $N$-acetyl-D-tryptophan & -3.3 & -19.0 & -15.5 \\
$\alpha$-chymotrypsin & $\beta$-naphthoate & -5.3 & -17.5 & -11.9 \\
$\alpha$-chymotrypsin & phenol & -3.1 & -13.5 & -10.4 \\
MOPC 315a) & dinitronaphthol & -6.5 & -20.2 & -13.7 \\
MOPC 315 & DNP-Lys & -8.1 & -16.6 & -8.4 \\
Anti-DNP antibody & DNP-Lys & -10.8 & -15.3 & -4.5 \\
& & & & \\
\hline
\end{tabular}

a) mouse plasmacytoma protein 


\section{COMPLEXATION OF AROMATIC HYDROCARBONS IN ORGANIC SOLVENTS}

The solubility properties of the macrobicyclic system 3 allowed investigations of the complexation of polycyclic arenes (perylene, pyrene, fluoranthene, naphthalene, and durene) in a wide range of organic solvents. [5] The geometry of the complexes of a given guest is very similar in all solvents and the geometry of the 3.pyrene complex is schematically depicted in Figure 2.

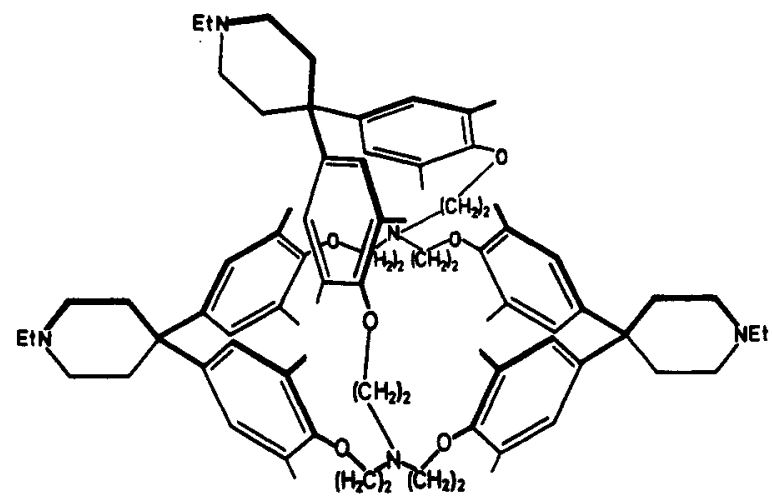

3

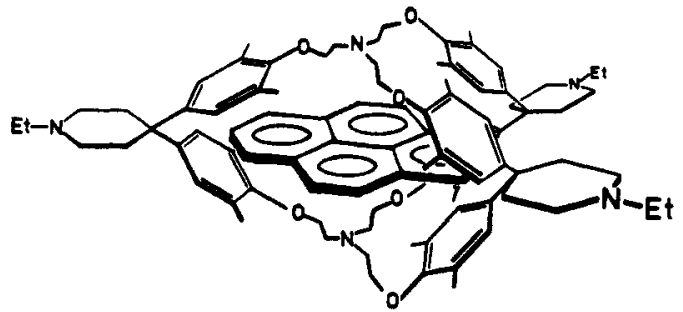

Fig 2. Schematic drawing of the geometry of the 3.pyrene complex in organic solvents

Table 3 displays the association constants of the 3.pyrene complex for a large variety of solvents. Binding in all solvents is considerably weaker than in aqueous solution. An impressive dependency of the complexation strength on the nature of the solvent is observed. The driving force for complexation decreases from $\Delta G^{\circ}=-6.4 \mathrm{kcal} \cdot \mathrm{mol}^{-1}$ in methanol $(303 \mathrm{~K})$ to $\Delta G^{0}=-1.3 \mathrm{kcal} \cdot \mathrm{mol}^{-1}$ in carbon disulfide. The strongest binding is observed in alcohols. Weaker but still considerable complexation occurs in dipolar-aprotic solvents, and the binding is weakest in chloroform, benzene, and carbon disulfide. The same solvent dependency of the complexation strength is also observed with other host-guest combinations. Since the geometry of the 3-pyrene complex is very similar in all solvents, the large decrease in binding strength upon changing from methanol to carbon disulfide cannot result from differences in attractive van der Waals interactions between host and guest in the complexes. Rather, solvation phenomena are responsible for the observed differences in complexation strength. This hypothesis is supported by the correlation of the free enthalpies of formation of the 3-pyrene complex with the empirical Dimroth-Reichardt solvent parameter $E_{\mathrm{T}}$ (Figure 3).

Table 3. $K_{\mathrm{a}}$ and $-\Delta G^{\circ}$ of the 3 pyrene complex in various organic solvents $(T=303 \mathrm{~K})$

\section{Solvent $\quad K_{\mathrm{a}}\left[\mathrm{L} \cdot \mathrm{mol}^{-1}\right] \quad-\Delta G^{\circ}\left[\mathrm{kcal} \cdot \mathrm{mol}^{-1}\right]$}

\begin{tabular}{lcl}
\hline Methanol & $4.4 \times 10^{4}$ & 6.4 \\
Ethanol & $2.5 \times 10^{4}$ & 6.1 \\
Acetone & $1.2 \times 10^{3}$ & 4.3 \\
$\mathrm{Me} 2 \mathrm{SO}$ & $6.9 \times 10^{2}$ & 3.9 \\
$\mathrm{DMF}$ & $1.2 \times 10^{2}$ & 2.9 \\
$\mathrm{THF}$ & 84 & 2.7 \\
$\mathrm{CHCl}_{3}$ & 43 & 2.3 \\
Benzene & 12 & 1.5 \\
$\mathrm{CS}_{2}$ & 9 & 1.3 \\
\hline
\end{tabular}

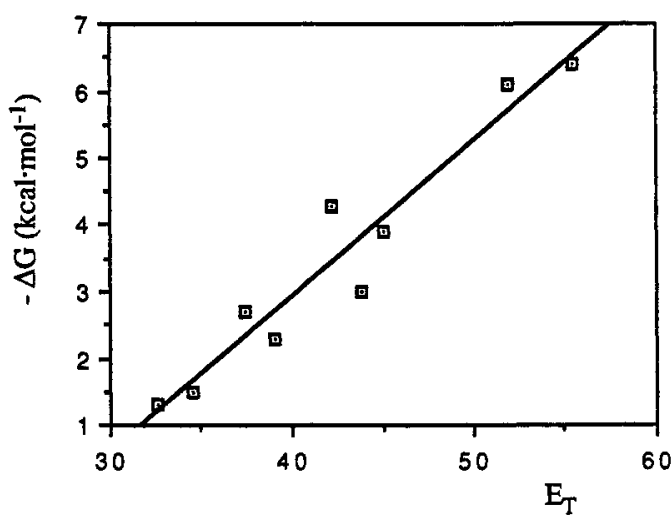

Fig 3. Free enthalpies of complexation $-\Delta G^{0}$ (kcal' $\mathrm{mol}^{-1}$ ) of the 3.pyrene complex in various organic solvents plotted against the empirical solvent polarity parameters $E_{\mathrm{T}}$ of these solvents.

The analysis of the observed solvent dependency shows that the complexation strength is dependent on the ability of the solvent molecules to compete effectively with the guest (pyrene) for the cavity of host 3 . The extent to which a solvent molecule can act as an efficient competitor of the guest for the cavity binding site is determined by its affinity to the cavity and by the cohesive interactions in the solvent. The weakest binding is observed in chloroform, 
benzene, and carbon disulfide. Experimental evidences for favorable interactions between these molecules and the binding site of 3 are available. The affinity of carbon disulfide for apolar binding sites has been observed independently by Cram et al.[22]. Specific aromatic solvent induced shifts (ASIS) in the ${ }^{1} \mathrm{H}$ NMR spectra of 3 in benzene indicated interactions of the benzene molecules with the cavity. A chloroform molecule enclosed in the cavity can form a $\mathrm{N} \cdot \cdot \mathrm{H}-\mathrm{CCl}_{3}$ hydrogen bond to the cryptand nitrogen atoms of 3 .

The cohesive interactions in a solvent determine the energy it costs for transferring a solvent molecule from the bulk into a host cavity. In our discussions of the binding properties of 1 and 2 above, we already mentioned that the large cohesive interactions between water molecules make the solvation of the apolar cavity of a host energetically unfavorable. The molar heat of vaporization $\Delta H^{0}$ vap [kcal $\mathrm{mol}^{-1}$ ] is a measure for the cohesive interactions in a given solvent. [23] If the $\Delta G^{\circ}$-values of Table 3 or those for the 3 perylene complex [5] are plotted against the molar heats of vaporization $\Delta H^{\circ}$ vap of the solvents, a significant correlation, analogous to the one shown in Figure 3, is obtained. The decrease in free binding energy upon changing from protic solvents to dipolar aprotic solvents and to benzene and carbon disulfide is parallel to the decrease in cohesive forces, expressed by the molar heats of vaporization. At smaller $\Delta H^{\circ}$ vap values, it becomes increasingly more favorable to transfer a solvent molecule for solvation from the bulk into the cavity. The solvents with lower $\Delta H^{\circ}$ vap values become increasingly more competitive with the guest for the host cavity.

The contributions of solvent to apolar binding demonstrated in the studies with hosts 1-3 clearly show that predictions of the stability of host-guest complexes based on computer-modeling can only be successful if specific solvation free energies are taken into account.

\section{CATION AND APOLAR COMPLEXATION BY A DITOPIC CYCLOPHANE HOST}

The binaphthyl building block 4 is an interesting ditopic unit with two distinctive geometric clefts. With a minor groove $\left(O \cdots O\right.$ distance $\approx 3.60 \AA$ at a dihedral angle about the chirality axis of $\left.\Theta=88^{\circ}\right)$ and a major groove $(0 \cdots O$ distance $\approx 7.05 \AA$ ), this unit has the potential for shaping two very different binding sites in macrocyclic receptors. The minor groove of binaphthyl units has been successfully incorporated into chiral cation binding receptors by Cram et al. [24] and Lehn et al.[25] Computer modeling in our laboratory led to the conclusion that efficient hosts for flat aromatic guests incorporate both an achiral or chiral aromatic spacer unit having an $\mathrm{O} \cdots \mathrm{O}$ distance of $\geq 6.5$ $\AA$ and a diphenylmethane type unit bridged by suitably sized aliphatic chains.[26] Hence, the major groove of 4 with its $0 \cdots$ O distance of $\approx 7.05 \AA$ should be ideal to shape an aromatic binding site in cyclophane hosts. To investigate the potential of 4 for shaping both an efficient cation binding site and an aromatic substrate binding site, we prepared the ditopic cyclophane host 5 .
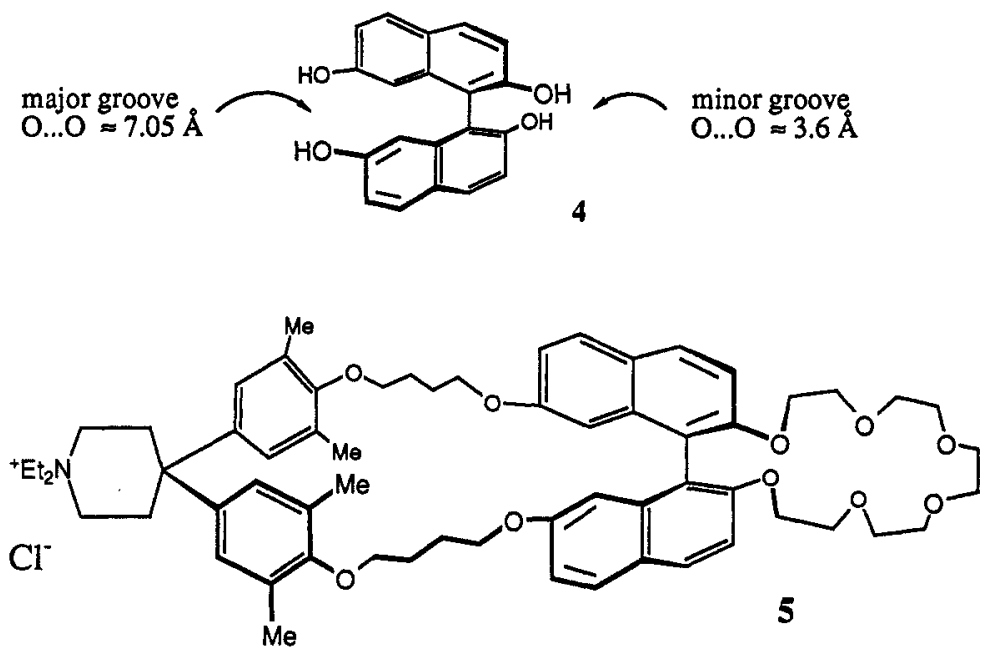

In $\mathrm{D}_{2} \mathrm{O}$ / methanol-d $(60: 40)$ at $293 \mathrm{~K}$, we observed by ${ }^{1} \mathrm{H}$ NMR the formation of stable complexes of 5 with a variety of naphthalene derivatives. The naphthalene guests take an axial position in the apolar binding site of 5 . With a free energy $\Delta G^{\circ}$ of $-4.90 \mathrm{kcal} \cdot \mathrm{mol}^{-1}$ for the complexation of 2-cyano-6-methoxynaphthalene (6) in this environment (Table 4), the apolar binding capability of 5 rivals that of bis(diphenylmethane)-hosts. If the methanol content of the solution is increased, the complexation of 6 is weakened. [27] Cation binding by 5 shows the opposite solvation dependency. In pure methanol- $d_{4}$, this ditopic host is a good binder of potassium cations; while in $\mathrm{D}_{2} \mathrm{O} /$ methanol-d $d_{4}(60: 40)$, complexation becomes very weak. ${ }^{[28]}$ Although the ${ }^{1} \mathrm{H}$ NMR spectra suggest considerable conformational changes of 5 both upon cation and/or naphthalene binding, the binding characteristics 
Table $4 . K_{\mathrm{a}}$ and $-\Delta G^{\circ}$ values for the complexes of host 5 with $\mathrm{K}^{+}$and with $6(T=293 \mathrm{~K})$

\begin{tabular}{lllll}
\hline & \multicolumn{5}{c}{$\mathrm{D}_{2} \mathrm{O}$ /methanol-d } \\
& $60: 40$ & $40: 60$ & $20: 80$ & $0: 100$ \\
& & & & \\
\hline Guest: $\mathrm{K}^{+}$ & & & & \\
$K_{\mathrm{a}}\left[\mathrm{L} \cdot \mathrm{mol}^{-1}\right]$ & $\approx 13$ & 89 & 274 & 1770 \\
$-\Delta G^{0}\left[\mathrm{kcal} \cdot \mathrm{mol}^{-1}\right]$ & $\approx 1.5$ & 2.61 & 3.27 & 4.35 \\
Guest: $6^{-}$ & & & & \\
$K_{\mathrm{a}}\left[\mathrm{L} \cdot \mathrm{mol}^{-1}\right]$ & 4510 & 441 & 142 & 24 \\
$-\Delta G^{0}\left[\mathrm{kcal} \cdot \mathrm{mol}^{-1}\right]$ & 4.90 & 3.52 & 2.88 & 1.85 \\
\hline
\end{tabular}

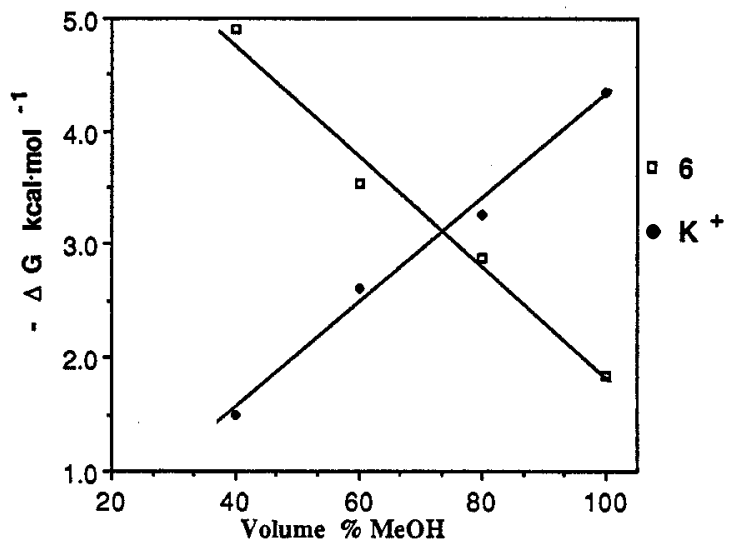

Fig 4. Opposite solvation characteristics of cation and apolar binding by 5

of the two recognition sites are almost independent of each other. In all solvent mixtures, the formation of a 1:1:1 host-guest ${ }_{1}$-guest 2 ) complex could be observed. Figure 4 summarizes the contrasting solvent characteristics of cation and apolar binding by cyclophane 5 . This host constitutes a ditopic receptor which can be switched from an efficient binder of neutral aromatics to a good host for potassium cations by changing the water-content of the methanol-water solvent mixture.

Acknowledgement This work was supported by the National Science Foundation and the Office of Naval Research.

\section{REFERENCES}

1. M. L. Bender and M.Komiyama, Cyclodextrin Chemistry, Springer, Berlin (1978).

2. K. Odashima, A. Itai, Y. Iitaka and K. Koga, J. Am. Chem. Soc., 102, 2504-2505 (1980).

3. F. Diederich, Angew. Chem., 100, 372-396 (1988); Angew. Chem. Int. Ed. Engl, 27, 362-386 (1988).

4. F. Diederich and K. Dick, I. Am. Chem. Soc., 106, 8024-8037 (1984).

5. F. Diederich, K. Dick and D. Griebel, I. Am. Chem. Soc, 108, 2273 - 2286 (1986).

6. S. B. Ferguson, E. M. Seward, F. Diederich, E. M. Sanford, A. Chou, P. Inocencio-Szweda and C. B. Knobler, J. Am. Chem. Soc. submitted for publication.

7. M. Hester, M. A. Uyeki and F. Diederich, Angew. Chem., submitted for publication.

8. C. Krieger and F. Diederich, Chem. Ber, 118, 3620-3631 (1985).

9. F. Diederich, K. Dick and D. Griebel, Chem. Ber., 118, 3588-3619 (1985).

10. S. B. Ferguson and F. Diederich, Angew, Chem, 28, 1127-1129 (1986); Angew, Chem. Int. Ed. Engl. $25,1127-1129$ (1986)

11. T. J. Shepodd, M. A. Petti and D. A. Dougherty, J. Am. Chem. Soc., 110, 1983-1985 (1988).

12. A. Leo, C. Hansch and D. Elkins, Chem. Rev., 71, 525-616 (1971).

13. M. R. Eftink and J. C. Harrison, Bioorg. Chem., 10, 388-398 (1981).

14. K. Harata, K. Tsuda, K. Uekama, M. Otagiri and F. Hirayama, J. Incl, Phenom, 6, 135-142 (1988)

15. W. Saenger, Angew. Chem., 22, 343-361 (1980); Angew. Chem. Int. Ed. Engl, 19, 344 - 362 (1980).

16. M. H. Abraham, J.Am. Chem. Soc., 104, 2085-2094 (1982)

17. A. Fersht, Enzyme Structure and Mechanism, pp 293-310, Freeman, New York (1985).

18. J. A. McCammon, P. G. Wolynes and M. Karplus, Biochemistry, 18, 927-942 (1979).

19. C. Tanford, The Hydrophobic Effect: Formation of Micelles and Biological Membranes, Wiley, New York (1980), 2nd ed.

20. C. Chothia, Nature, 248, 338-339 (1974)

21. The biological recognition data are taken from: R. L. Biltonen and N. Langerman, Methods Enzymol., 61, 287-318 (1979).

22. D. J. Cram, K. D. Stewart, I. Gold'verg and K. N. Trueblood, J. Am. Chem. Soc, 107, 2574-2575 (1985).

23. I. Tabushi, Y. Kiyosuke, T. Sugimoto and K. Yamamura, J. Am. Chem. Soc, 100, 916-919 (1978).

24. D. J. Cram and J. M. Cram, Science, 183, 803-809 (1974).

25. J.-M. Lehn, J. Simon and A. Moradpour, Helv. Chim. Acta, 61, 2407-2418 (1978).

26. R. Dharanipragada, S. B. Ferguson and F. Diederich, J. Am. Chem. Soc., 110, 1679-1690 (1988).

27. H.-J. Schneider, K. Philippi and J. Pöhlmann, Angew. Chem., 96, 907-909 (1984); Angew. Chem. Int. Ed. Engl. 23, 908-910 (1984).

28. R. M. Izatt, R. E. Terry, D. P. Nelson, Y. Chan, D. J. Eatough, J. S. Bradshaw, L. D. Hansen and J. J. Christensen, J.Am. Chem. Soc, 98, 7626-7630 (1976). 